\title{
Developmental Competence of Early Stage Porcine Embryos Cultured in Medium with D ifferent Energy Substrate in vitro
}

\section{Ni Wayan Kurniani Karja ${ }^{*}$, Mokhamad Fahrudinand Kazuhiro Kikuchi ${ }^{3}$}

1. Department of Reproduction and Obstetric, Faculty of Veterinary Medicine, Gadjah Mada University, Yogyakarta55281, Indonesia.

2. Faculty of Veterinary Medicine, Bogor Agricultural University, Bogor 16680, Indonesia.

3. Department of Animal Sciences, Reproductive Biology Research Unit, National Institute of Agrobiological Sciences, Tsukuba, Ibaraki 305-8602, Japan.

\begin{abstract}
A bstract
To elucidate the effect of energy requirement during the early embryonic development on their developmental ability to develop to blastocyst stage, in vitro fertilized (IVF) porcine one-cell embryos were cultured in modified North Carolina State University (NCSU)-37 supplemented with different energy substrate. Result indicated that the deavage rate of embryos in Pyr-Lac and Gluc-Pyr-Lact groups was significantly higher than in those in Gluc group and Gluc-Rib group ( $P<0.05$ ). At Day 6 of culture, the highest proportion of embryos devel op to the blastocyst stage was obtained in the presence of pyruvate-lactate only. In the medium with glucose, the addition of pyruvate-lactate or ribose slightly increased the proportion of embryos develop to the blastocyst stage, however the value were not significantly different form those obtained in the presence of glucose only. The mean cell number in blastocysts derived from Pyr-Lac and Gluc-Pyr-Lact groups were significantly higher than those in the Gluc group $(P<0.05)$. Theseresults indicated that the presence of glucoseonly, as energy substrate, during thefirst 2 days of in vitro culture (IVC) caused a decrease in development of in vitro produced (IVP) porcineembryos to theblastocyst stage and mean cell number in blastocysts.
\end{abstract}

Keywords: porcineembryos-energy substrate-in vitroculture

\section{Introduction}

The development of culture system to support early embryonic development is important for study of the factor that are involved as well as for practical use in such biotechnologiesasin vitro fertilization (IVF), nuclear transplantation and genetransfer by DNA microinjection. Embryos from most species exhibit less than optimal development in vitro and may 'block'

*Corresponding author: A ris Haryanto, Department of Biochemistry, Faculty of Veterinary Medicine, Gadjah Mada University, Yogyakarta. Jl. Olag Raga, Karangmalang, Yogyakarta 55281, Indonesia. Telp. 62-274-560865. E-mail: arisharyanto@yahoo.com during development at specific cell stages (Bavister, 1979). Thus, the culture medium or environmental conditions used for in vitro culture must be inferior for supporting normal development compare than in vivo condition. In vitro culture conditions deviate from in vivo situations in many respects, but one of the critical factors appears to be the energy substrates present in culturemedium for the production of energy and for the synthesis of a variety of complex molecules, such us glucose, pyruvate and lactate (Rieger et al., 1992).

It is well known that metabolic activity and substrates preferences of embryos appear to change between early and late 
cleavages with elevated glucose and oxygen consumption as they approach cavitation (Houghton et al., 1996). Most mammalian embryos need pyruvate as a substrate of energy in the early stages of development (Rieger et al., 1992; Gardner et al., 1993). Pyruvate metabolism was relatively high at the two- and four-cell stages, declined to a minimum at the compacted morula stage, and then increased at blastulation. Glucose metabolism via glycolysis, on the other hand, increased between the eight-cell and 16-cell stage, and continued to increase up to the blastocyst stage (Rieger et al., 1992). Similarly, glucose uptake by sheep embryos is low before the 16-cell stage and increases steadily thereafter (Gardner et al., 1993). Unlike cattle embryos, pyruvate uptake by sheep embryos is relatively constant up to the morula stage and then increases significantly through the blastocyst stage (Thompson et al., 1992; Gardner et al., 1993). In the mouse, although the zygote and early cleavagestageembryo cannot useglucoseas the sole substrate of energy, the blastocysts exhibit a large capacity to utilize glucose both oxidatively and through aerobic glycolysis (Chatot et al., 1989). In pigs, it has been reported that the embryos consumed glucose and produced lactate at all stages of development, utilization of glucose was low during the early cleavage stages, increased slightly at the morula stage and reached a peak at the early blastocyst stage (Sturmey and Leese, 2003). Therefore, the uptake and metabolism of energy substrate such as, glucose and pyruvate are appears to be developmentally related.

Although IVF and embryo production using in vitro matured oocytes have been successful and many laboratories have tried to overcome inadequacies of their IVC system by supplementing culture medium with various energy substrate or using stepwise culture system with components that may be advantageous for different stages of embryo development (Petters and Wells 1993; Gandhi et al., 2001; Kikuchi et al., 2002; Karja et al., 2004a, 2004b), their developmental ability and the quality of blastocyst yielded are also still low compared to in vivo-derived blastocyst. Therefore, the purpose of this study was to observe developmental ability of early stage porcine embryos after culturing in medium with different energy substrate. We have focused specifically the experiment on the first 2 days of culture, early stage of embryo development, since the first 2 days of culture in porcine embryos seem to have a critical effect on further development (Kikuchi et al., 2002), at period when the activation of embryonic genome occurs. In the suboptimal in vitro culture conditions, embryos are blocked in developmental or slowing down in cleavages at thestage when the activation of embryonic genome occurs (Telford et al., 1990)

\section{M aterials and M ethods 0 ocyte collection and in vitro maturation (IVM)}

Collection and IVM of porcinefollicular oocytes were performed according to Kikuchi et al. (2002). Briefly, ovaries from prepubertal cross-bred gilts (Landrace $X$ Large White) were collected at a local slaughterhouseand carried to thelaboratory in Dulbecco's PBS (Nissui Pharmaceutical Co. Ltd) at 35-37 8C within $1 \mathrm{~h}$. Cumulus-oocyte complexes (COCs) were collected by scraping of 3-6 mm follicles in a collection medium consisting of Medium 199 (with Hanks' salts; Sigma Chemical Co.) supplemented with $10 \%$ fetal bovine serum (Gibco and Invitrogen Corp.), 20mM HEPES (Dojindo Laboratories), and antibiotics (100 units/ $\mathrm{ml}$ penicillin $\mathrm{G}$ potassium (Sigma) and $0.1 \mathrm{mg} / \mathrm{ml}$ streptomycin sulfate (Sigma)). Maturation culturewas performed in a modified North Carolina State University (NCSU)-37 solution (Petters and 
Wells, 1993) containing 10\% (v/ v) porcine follicular fluid, $0.6 \mathrm{mM}$ cysteine, $1 \mathrm{mM}$ dibutyryl CAMP (dbcAMP, Sigma), 10 $\mathrm{IU} / \mathrm{ml}$ eCG (PMS 1000 Tani NZ, Nihon Zenyaku Kogyo), and $10 \mathrm{lU} / \mathrm{ml}$ hCG (Puberogen, $500 \mathrm{U}$, Sankyo) in a four-well dishes (N unclon Multidishes) for $22 \mathrm{~h}$ in an atmosphere of 5\% CO2, 5\%O2, and 90\% N2 at $39^{\circ} \mathrm{C}$. COCs were subsequently cultured in maturation medium without dbcAMP and hormones for an additional $24 \mathrm{~h}$ under thesameatmosphere.

\section{In vitro fertilization (IVF)}

Spermatozoa were thawed and preincubated for $15 \mathrm{~min}$ at $38.5^{\circ} \mathrm{C}$ in a tissue culturemedium (TCM) 199with Earle's salts (Gibco) adjusted to pH 7.8. A portion (10 $\mu$ l) of preincubated spermatozoa was introduced into $90 \mu \mathrm{l}$ of fertilization medium containing 10-20 matured COCs. Fertilization medium consists of $90 \mathrm{mM}$ $\mathrm{NaCl}, 12 \mathrm{mM} \mathrm{KCl}, 25 \mathrm{mM} \mathrm{NaHCO}_{3}, 0.5 \mathrm{mM}$ $\mathrm{NaH}_{2} \mathrm{PO}_{4}, 0.5 \mathrm{mM} \mathrm{MgSO}, 10 \mathrm{mM}$ sodium lactate, $3 \mathrm{mg} / \mathrm{ml}$ bovine serum albumin (BSA; Fatty acid free, Sigma), $5 \mathrm{mM}$ caffeine (Sigma) and $50 \mu \mathrm{g} / \mathrm{ml}$ gentamicin. The final sperm concentration was adjusted to $1 \mathrm{x}$ $10 / \mathrm{ml}$. The oocytes were co-incubated with spermatozoa for $3 \mathrm{~h}$. And then, the inseminated oocytes weredenuded from the cumulus cells and attached spermatozoa by mechanical pipetting and transferred to culture medium to which they had been designed, as bellow.

\section{Embryo culture}

The basic medium used for embryo culture was glucose-free NCSU-37 containing $4 \mathrm{mg} / \mathrm{ml} \mathrm{BSA}$ and $50 \mu \mathrm{M}$ bmercaptoethanol (IVC medium). To observe developmental ability o developmental ability of early stage porcine embryos after culturing in medium with different energy substrate for the first 2 days of culture, presumptive zygotes were cultured from
Days 0 (the day of insemination was defined as day 0 ) to 2 in IVC medium supplemented with (1) $5.5 \mathrm{mM}$ glucose (Gluc group), (2) $0.17 \mathrm{mM}$ sodium pyruvate and $2.73 \mathrm{mM}$ lactate (Pyr-Lact group), (3) $5.5 \mathrm{mM}$ glucose, $0.17 \mathrm{mM}$ pyruvate and $2.73 \mathrm{mM}$ lactate (Gluc-Pyr-Lact group), or (4) $5.5 \mathrm{mM}$ glucose and $5.5 \mathrm{mM}$ Ribose-5 phosphate (Gluc-Rib group). Subsequently, embryos in all groups were cultured until Day 6 in IVC medium supplemented with $5.5 \mathrm{mM}$ glucose only. Culturewas performed at $38.5^{\circ} \mathrm{C}$ and $5 \% \mathrm{CO}_{2}$ under $5 \% \mathrm{O}_{2}$ and $90 \% \mathrm{~N}_{2}$.

\section{Embryos evaluation}

For examination of the embryo development, on Day 6 all embryos in all groups were fixed and permeabilized for 15 min at room temperature with $3.7 \%$ paraformaldehyde in PBS containing 1\% (v/ v) Triton X-100 (Sigma), and then placed in PBS supplemented with 0.3̈̈ (w/v) polyvinylpyrrolidone (PVP; Sigma) for 15 min at room temperature. Theembryos were placed on a microslidein a drop of mounting medium consisting of $90 \%(\mathrm{v} / \mathrm{v})$ glycerol containing $1.9 \mu \mathrm{M}$ bis-benzimide (Hoechst 33342; Cal biochem; EMD Biosciences, Inc. ). A coverslip was placed on top of the embryos and the staining of the nuclei was examined with a fluorescence microscope (BX 51; Olympus). The numbers of cleaved embryos including the stage of embryo development, blastocysts formation rate, and the cell number in blastocyst were recorded.

\section{Statistical Analysis}

All percentage data were subjected to arc-sine transformation before statistical analysis. Then all data, including the arcsine-transformed percentages of blastocyst formation and the data on mean number of cells in the blastocyst were subjected to ANOVA by a post hoc, Fisher's protected least significant difference test 
(PLSD test) using the STATVIEW (Abacus Concepts Inc., Berkeley, CA, USA ) program. Data were expressed as means \pm SEM. Differences at $\mathrm{P}<0.05$ were considered significant.

\section{Results and D iscussion}

The results of in vitro cleavage and development of porcine zygotes to the blastocyst stage and total cell number in blastocyst are shown in Table 1. When the embryos were cultured in the medium without any energy substrate, no cleavage and development to blastocyst stage were observed. In the medium supplemented with pyruvate-lactate only (Pyr-Lac group) or together with glucose (Gluc-Pyr-Lact groups), the proportion of embryos that cleaved at 48 hours after IVC was significantly higher than in medium with glucose only (Gluc group) or together with ribose (Gluc-Rib group) $(P<0.05)$. At Day 6 of IVC, the highest proportion of embryos develop to the blastocyst stage was obtained in the presence of pyruvate-lactate only. In the medium with glucose, the addition of pyruvate and lactate or ribose slightly increased the proportion of embryos develop to blastocyst stage, but the values were not significantly different from those obtained in gluc-group.

Table 1. Developmental competence of porcine embryos cultured in medium with different energy substrates during early embryonic development

\begin{tabular}{lcccc}
\hline Groups & $\begin{array}{c}\text { No. of } \\
\text { oocytes }\end{array}$ & $\begin{array}{c}\text { Mean } \pm \text { SEM } \\
\text { (No.) of cleaved } \\
\text { examined }\end{array}$ & $\begin{array}{c}\text { Mean } \pm \text { SEM } \\
\text { (No.) of embryos } \\
\text { embryos }\end{array}$ & $\begin{array}{c}\text { Mean } \pm \text { SEM } \\
\text { of total cell } \\
\text { developed }\end{array}$ \\
\hline Gluc & 120 & $40.8 \pm 2.8(49)^{\circ}$ & $13.3 \pm 1.4(16)^{\circ}$ & $32.6 \pm 2.7^{\circ}$ \\
\hline Pyr-lact & 115 & $68.0 \pm 4.9(80)^{\circ}$ & $24.2 \pm 3.1(29)^{\circ}$ & $40.8 \pm 2.8^{\circ}$ \\
\hline Gluc-Pyr-Lact & 147 & $64.7 \pm 4.2(97)^{\circ}$ & $19.7 \pm 3.4(23)^{-\infty}$ & $40.7 \pm 2.9^{\circ}$ \\
\hline Gluc-Rib & 110 & $46.7 \pm 7.8(52)^{\circ}$ & $21.3 \pm 4.7(24)^{*}$ & $35.5 \pm 2.2^{*}$ \\
\hline None & 125 & 0 & 0 & \\
\hline
\end{tabular}

Four replicatetrial swerecarried out. Values areexpressed as means \pm SEM

ab values with different superscript letters are significantly different $(p<0.05)$

As shown in Table 1, the mean cell number in blastocysts derived from Pyr-Lac and Gluc-Pyr-Lactgroups weresignificantly higher than those in the Gluc group $(P<$ 0.05). Developed porcine embryos at blastocyst stage and blastocyst with Hoechst-labeled nuclei at Day 6 of culture wererepresented in Figure 1.

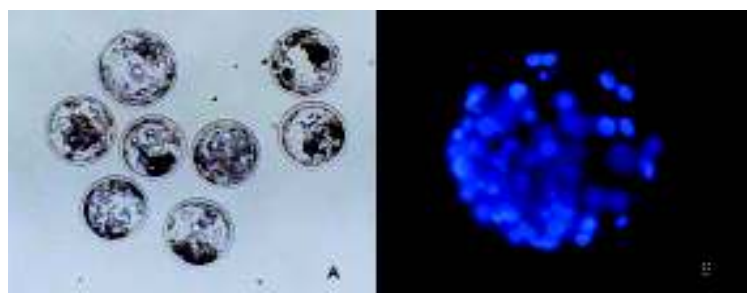

Figure 1. Developed porcine embryos at blastocyst stage (A) and blastocyst with Hoechst-labeled nuclei (B) at Day 6 of culture (original magnification 100x).

The types and concentrations of energy substrates added to a culture medium alter metabolic profiles, development and quality of embryos. The results from this study has demonstrated that when porcine IVM-IVF oocytes were cultured in IVC medium supplemented with pyruvateand lactate for the first 2 days and then cultured in the medium containing glucose for subsequent 4 days, the rate of blastocyst formation the cell number in blastocysts were higher as compared with that of glucose supplement for the first 2 days. Although porcine embryos has been reported areable to utilize glucose throughout their development, results from the present study indicate that glucose alone as energy substrate during early stage of porcine embryos development could not support development of the embryos to the blastocyst stage. The lack of responseto theglucosein thisstudy could be explained as thelimited ability of early stage 
porcineembryos to utilizeglucose (Sturmey and Leese, 2003) or the embryos use only a fraction of the glucose that they take up (Swain et al., 2002). Porcine embryos appear to behavesimilar fashion metabolically as do other domestic livestock species embryos, with limited glucose utilization before the morula stage and a heavy reliance on glycolysis during blastocoele formation (Flood and Wiebold, 1988; Rieger et al., 1992; Gandhi et al., 2001). On the other hand, culturing porcine embryos in a combined medium that provided $5.5 \mathrm{mM}$ glucose, pyruvate and lactate, or glucose combining with $5.5 \mathrm{mM}$ ribose 5-phosphate, the end product of glucose metabolism through pentose phosphate pathway (PPP), as energy substrates for the first 2 days and followed by culture in medium with glucose $(5.5 \mathrm{mM})$ the next 4 days slightly improved blastocyst development and cell number in blastocysts compared with those cultured for $144 \mathrm{~h}$ in medium with glucose. Taken together, these results clearly indicate that glucosein IVC medium for the first 2 days of culture is detrimental to the development of embryos, while pyruvate and lactate appear to be important energy substrates for both early embryonic development in vitro and for improving the number of cells in blatocysts. Our observation confirms the previous reports, which reported that pyruvate and lactate sseem to be predominant energy substrates for the first cleavage division for porcine embryos (Kikuchi, 2002, Kim et al., 2004). Pyruvate and lactate may also provide the embryos with more suitable conditions for cellular oxidation reduction equilibrium, resulting in viable embryo growth and development (Thompson et al. 1993). Butcher et al. (1998) recently suggested that pyruvate might be converted to alanine and may play a role in removing ammonia from embryos. A nother possible function for pyruvate is to protect embryos against oxidative stress as suggested by Leese(1991).

Glucose is usually included in standard tissueculturemedia at a concentration of 5.5 $\mathrm{mM}$, on the basis of its concentration in serum. This is much higher compared than in the oviduct, in which in vivo porcine embryos are exposed (0.17 mmol glucose $\left.\mathrm{l}^{2}\right)$ (N ichol et al ., 1992). The del eterious effects of glucose on embryo development in vitro have been reported. The most likely reason that glucoseaffects the development of early stage embryo, therefore, is that one or more metabolites generated by the PPP leads to a failure in development of the embryos. One possibility is that NADPH, although general ly regarded as protectiveagainst free radical damage (Salvemini et al., 1999), is produced in such quantities that its reoxidation actually generates oxygen free radicals, an occurrence that is well documented in some cell types (Babior, 2000). The use of glucose as an energy substrate results in the production of reactive oxygen species (ROS), particularly the superoxide anion and the hydroxyl radical. Production of superoxide anion and $\mathrm{H}_{2} \mathrm{O}_{2}$ via glucose metabolism through PPP has been described on rabbit blastocyst (Manes and Lai, 1995). Reactive oxygen species are highly active electron acceptors, able to strip electrons from other molecules that, in turn, becomefreeradicals. Hydrogen peroxide $\left(\mathrm{H}_{2} \mathrm{O}_{2}\right)$ is not a radical per se, but is a product of $\mathrm{O}_{2} \mathrm{E}$ ?and metal ion catalysis. However, both $\mathrm{H}_{2} \mathrm{O}_{2}$ and $\mathrm{O}_{2} \mathrm{E}$ can form the extremely reactive OHÉ. The overgeneration of intracellular ROS during culture of mammalian embryos in vitro is generally thought to be detrimental to embryo development (Johnson and NasrEsfahani, 1994; Guerin et al., 2001). 


\section{Acknowledgements}

The authors wish to thank Dr. Takashi Nagai for his helpful suggestions in the preparation of this manuscript. Thanks also to the staff members of the Department of Animal Sciences, Reproductive Biology Research Unit, National Institute of Agrobiological Sciences, for their support and comments. Thisstudy was supported by a grant-in-aid for Japanese Society for the Promotion of Science Postdoctoral Fellowship for Foreign Researchers ( $P$ 04588).

\section{References}

Babior, B.M., 2000. Phagocytes and oxidative stress. A m. J. M ed., 109:33-44.

Bavister, B.D., 1979. Fertilization of hamster eggs in vitro at sperm:egg ratios close to unity. J. Exp. Z ool., 210:259-264.

Butcher, L., Coates A., Martin, K.L., Rutherford, A.J. and Leese, H.J., 1998. Metabolism of pyruvate by the early human embryo. Biol. Reprod., 58:10541056.

Chatot, C.L., Ziomek, C.A., Bavister, B.D., Lewis, J.L. and Torres, I., 1989. An improved culture medium supports development of random-bred 1-cell mouse embryos in vitro. J. Reprod. Fertil. 86:679-688.

Flood, M.R. and Wiebold, J.L., 1988. Glucose metabolism by preimplantation pig embryos. J. Reprod. F ertil. 84:7-12.

Gandhi, A.P., Lane, M., Gardner, D.K. and Krisher, R.L., 2001. Substrate utilization in porcine embryos cultured in NCSU23 and G1.2/ G2.2 sequential culture media. $\mathrm{M}$ ol Reprod D ev. 58:269-275.

Gardner, D.K., Lane, M., and Batt, P., 1993. Uptake and metabolism of pyruvate and glucose by individual sheep preattachment embryos developed in vivo. M ol. Reprod. D ev., 36:313-319.

Guerin, P., El Mouatassim, S. and Menezo, Y., 2001. Oxidative stress and protection against reactive oxygen species in the pre-implantation embryo and its surroundings. Hum. Reprod. U pdate., 7:175-189.

Houghton, F.D., Thompson, J.G., Kennedy, C.J. and Leese, H.J., 1996. Oxygen consumption and energy metabolism of the early mouse embryo. Mol. Reprod. D ev., 44:476-485.

Johnson, M.H., and Nasr-Esfahani, M.H., 1994. Radical solutions and cultural problems: could free oxygen radicals be responsible for the impaired development of preimplantation mammalian embryos in vitro? Bioessays. 16:31-38.

Karja, N.W.K., Medvedev, S., Onishi, A., Fuchimoto, D., Iwamoto, M., Otoi, T. and Nagai, T., 2004a. Effect of replacement of pyruvate/ lactate in culture medium with glucose on preimplantation development of porcine embryos in vitro. J. Reprod. Dev., 50:587-592.

Karja, N.W. K., Wongsrikeao, P., Murakami, M., Agung, B., Fahrudin, M., Nagai, T. and Otoi, T., 2004b. Effects of oxygen tension on the development and quality of porcine in vitro fertilized embryos. Theriogen ology. 62:1585-1595.

Kikuchi, K., Onishi, A., Kashiwazaki, N., Iwamoto, M., N oguchi, J., Kaneko, H., Akita, T. and Nagai, T., 2002. Successful piglet production after transfer of blastocysts produced by a modified in vitro system. Biol. Reprod., 66:1033-1041.

Kim, H.S., Lee, G.S., Hyun, S.H., Lee, S.H., Nam, D.H., Jeong, Y.W., Kim, S., Kang, S.K., Lee, B.C. and Hwang, W.S., 2004. Improved in vitro development 
of porcine embryos with different energy substrates and serum. Theriogenology. 61:1381-1393.

Leese, H.J., 1991. Metabolism of the preimplantation mammalian embryo. 0 xfRev. Reprod. Biol. 13:35-72.

Manes, C., and Lai, N.C., 1995. Nonmitochondrial oxygen utilization by rabbit blastocysts and surface production of superoxide radicals. J. Reprod. Fertil. 104: 69-75.

Nichol, R., Hunter, R.H., Gardner, D.K., Leese, H.J., and Cooke. G.M., 1992. Concentrations of energy substrates in oviductal fluid and blood plasma of pigs during the peri-ovulatory period. J. Reprod. Fertil., 96:699-707.

Petters, R.M., and Wells, K.D., 1993. Culture of pig embryos. J. Reprod. Fertil. Suppl. 48:61-73.

Rieger, D., 1992. Relationship between energy metabolism and development of early mammalian embryos. Theriogenology. 37:75-93.

Salvemini, F., Franze, A., Iervolino, A., Filosa, S., Salzano, S., and Ursini. M.V.,1999. Enhanced glutathione levels and oxidoresistance mediated by increased glucose-6-phosphate dehydrogenase expression. J. Biol. Chem., 274:2750-2757.

Sturmey, R.G., and Leese, H.J., 2003. Energy metabolism in pig oocytes and early embryos. Reproduction. 126:197-204.

Swain, J.E., Bormann, C.L., Clark, S.G., Walters, E.M., Wheeler, M.B., Krisher, R.L., 2002. Use of energy substrates by various stage preimplantation pig embryos produced in vivo and in vitro. Reproduction. 123:253-260.

Telford, N.A., Watson, A.J. and Schultz, G.A., 1990. Transition from maternal to embryoniccontrol in early mammalian development: a comparison of several species. M ol. Reprod. D ev., 26:90-100.

Thompson, J.G.E., Simpson, A.C., Pugh, P.A., and Tervit, H.R.,1992. Requirements for glucose during in vitro culture of sheep preimplantation embryos. M ol. Reprod. D ev., 31:253257.

Thompson, J.G., Bell, A.C., Pugh, P.A. and Tervit, H.R., 1993. Metabolism of pyruvate by pre-elongation sheep embryos and effect of pyruvate and lactate concentrations during culture in vitro. R eprod. Fertil. D ev., 5:417-23. 